\title{
Review Article \\ Studies of Backward Particle Production with a Fixed-Target Experiment Using the LHC Beams
}

\author{
Federico Alberto Ceccopieri \\ IFPA, Université de Liège, Allée du 6 Août, 4000 Liège, Belgium \\ Correspondence should be addressed to Federico Alberto Ceccopieri; federico.alberto.ceccopieri@cern.ch
}

Received 19 March 2015; Revised 28 May 2015; Accepted 17 June 2015

Academic Editor: Jean-Philippe Lansberg

Copyright (C 2015 Federico Alberto Ceccopieri. This is an open access article distributed under the Creative Commons Attribution License, which permits unrestricted use, distribution, and reproduction in any medium, provided the original work is properly cited. The publication of this article was funded by $\mathrm{SCOAP}^{3}$.

\begin{abstract}
The foreseen capability to cover the far backward region at a Fixed-Target Experiment using the LHC beams allows one to explore the dynamics of the target fragmentation in hadronic collisions. In this report we briefly outline the required theoretical framework and discuss a number of studies of forward and backward particle production. By comparing this knowledge with the one accumulated in Deep Inelastic Scattering on target fragmentation, the basic concept of QCD factorisation could be investigated in detail.
\end{abstract}

\section{Introduction}

In hadronic collisions a portion of the produced particle spectrum is characterised by hadrons carrying a sizeable fraction of the available centre-of-mass energy, the so-called leading particle effect. It is phenomenologically observed that for such hadrons their valence-parton composition is almost or totally conserved with respect to the one of initial-state hadrons [1]. In $p p$ collisions, for example, protons, neutrons, and lambdas show a significant leading particle effect. For such processes, the production cross section peaks at very small transverse momenta with respect to the collision axis, a regime where perturbative techniques cannot be applied, giving insight into nonperturbative aspects of $\mathrm{QCD}$ dynamics in high energy collisions.

Quite interestingly, the leading particle effect has been observed in Semi-Inclusive Deep Inelastic Scattering (SIDIS). At variance with the hadronic processes mentioned above, such a process naturally involves a large momentum transfer. The presence of a hard scale enables the derivation of a dedicated factorisation theorem [2-5] which ensures that QCD factorisation holds for backward particle production in DIS. The relevant cross sections can then be factorised into perturbatively calculable short distance cross sections and new distributions, fracture functions, which simultaneously encode information both on the interacting parton and on the spectator fragmentation into the observed hadron. Despite of being nonperturbative in nature, their scale dependence can be calculated within perturbative QCD [6]. The factorisation theorem [2-5] guarantees that fracture functions are universal distributions, at least in the context of SIDIS.

Detailed experimental studies of hard diffraction at HERA have shown to support the hypothesis of QCD factorisation and evolution inherent the fracture function formalism. Furthermore, they led to a quite accurate knowledge of diffractive parton distributions [7-11], a special case of fracture functions in the very backward kinematic limit. For particles other than protons, proton-to-neutron fracture functions have been extracted from a $\mathrm{PQCD}$ analysis of forward neutron production in DIS in [12]. A set of proton-tolambda fracture functions has been obtained by performing a combined pQCD fit to a variety of semi-inclusive DIS lambda production data in [13].

As theoretically anticipated in $[2-4,14,15]$ and experimentally observed in hard diffraction in $p \bar{p}$ collisions at Tevatron [16, 17], QCD factorisation is violated for fracture functions in hadronic collisions. On general grounds, it might be expected, in fact, that the dynamics of target-remnants hadronisation is affected by the coloured environment resulting from the scattering in a rather different way with respect to the Deep Inelastic Scattering case.

Nonetheless, the tools mentioned above allow us to investigate quantitatively particle production mechanisms in 
the very backward and forward regions, to test the concept of factorisation at the heart of QCD, and to study the dependencies of factorisation breaking upon the species and the kinematics of the selected final state particle.

This physics program could be successfully carried on at a Fixed-Target Experiment using the LHC beams [18]. Novel experimental techniques are, in fact, available to extract beam-halo protons or heavy ions from LHC beams without affecting LHC performances. Such a resulting beam would be then impinged on a high-density and/or long-length fixed target, guaranteeing high luminosities. Furthermore, most importantly for the physics program to be discussed here, the entire backward hemisphere (in the centre-of-mass system of the collision) would be accessible with standard experimental techniques allowing high precision studies of the target fragmentation. Although measurements of particle production in the very forward region (close to the beam axis) might be challenging experimentally due to the high particle densities and large energy flow, the installation of dedicated detectors, like forward neutron calorimeters and/or proton taggers, could further broaden the physics program outlined above giving access to the beam fragmentation region.

The paper is organised as follows. In Section 2 we first give a brief theoretical introduction on the fracture functions formalism and to higher order corrections to the semiinclusive Drell-Yan process. In Section 3 we outline different analyses which could be performed at AFTER@LHC with special focus on single hard diffraction. In Section 4 we summarise our results.

\section{Collinear Factorisation Formula}

Fracture functions, originally introduced in DIS, do depend on a large momentum transfer. Therefore, in order to use them in hadronic collisions, a hard process must be selected. We consider here the semi-inclusive version of the Drell-Yan process:

$$
H_{1}\left(P_{1}\right)+H_{2}\left(P_{2}\right) \longrightarrow H(h)+\gamma^{*}(q)+X
$$

in which one hadron $H$ is measured in the final state together with a Drell-Yan pair. In such a process the high invariant mass of the lepton pair, $q^{2}=Q^{2}$, allows the applicability of perturbative QCD, while the detected hadron $H$ can be used, without any phase space restriction, as a local probe to investigate particle production mechanisms.

The associated production of a particle and a Drell-Yan pair in terms of partonic degrees of freedom starts at $\mathcal{O}\left(\alpha_{s}\right)$. One of the contributing diagrams is depicted in Figure 1.

Assuming that the hadronic cross sections admit a factorisation in terms of long distance nonperturbative distributions and short distance perturbative calculable matrix elements for the partonic process $i\left(p_{1}\right)+j\left(p_{2}\right) \rightarrow l(k)+$ $\gamma^{*}(q)$, predictions based on perturbative QCD are obtained convoluting the relevant partonic subprocess cross sections, $d \widehat{\sigma}^{i j \rightarrow l \gamma^{*}}$, with parton distribution functions, $f_{i / H_{1}}$ and $f_{j / H_{2}}$,

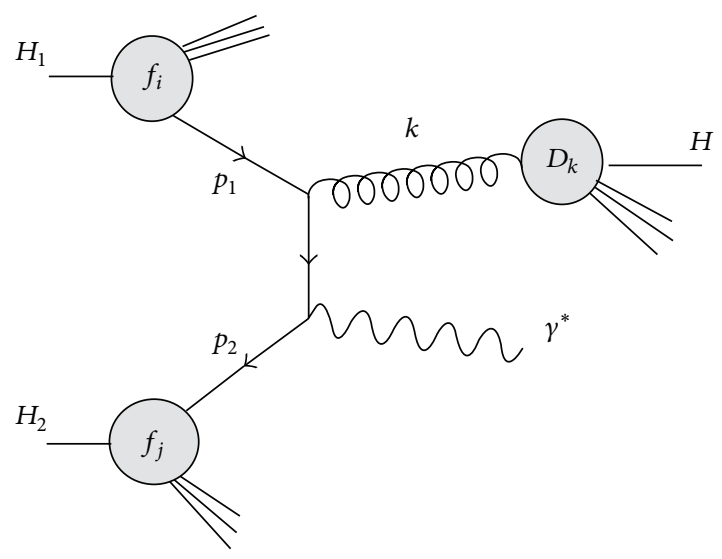

FIGURE 1: Example of diagram contributing to hadron production in the central fragmentation region to order $\mathcal{O}\left(\alpha_{s}\right)$ in (2).

and fragmentation functions, $D^{H / l}$. The hadronic cross section, at centre of mass energy squared $s=\left(P_{1}+P_{2}\right)^{2}$, can be symbolically written as $[19,20]$

$$
\begin{aligned}
& \frac{d \sigma^{H, C,(1)}}{d Q^{2} d z} \propto \sum_{i, j, l} \int \frac{d x_{1}}{x_{1}} \int \frac{d x_{2}}{x_{2}} \int \frac{d \rho}{\rho} f_{i}^{[1]}\left(x_{1}\right) f_{j}^{[2]}\left(x_{2}\right) \\
& \cdot D^{H / l}\left(\frac{z}{\rho}\right) \frac{d \widehat{\sigma}^{i j \rightarrow l \gamma^{*}}}{d Q^{2} d \rho},
\end{aligned}
$$

where the convolutions are over the momentum fractions of the incoming and outgoing partons. The partonic indices $i, j$, and $l$ in the sum run on the available partonic subprocesses. The superscripts label the incoming hadrons and the presence of crossed terms is understood. This type of factorised hadronic cross section is expected to hold for hadrons produced at sufficiently high transverse momentum and it is widely and successfully used to compute cross sections for large momentum transfer processes in hadronic collisions. The Lorentz-invariant variable $z$ in (2) is defined by

$$
z=\frac{2 h \cdot\left(P_{1}+P_{2}\right)}{s} \equiv \frac{2 E_{H}^{*}}{\sqrt{s}} .
$$

In the hadronic centre-of-mass frame, where the second identity holds, $z$ is just the observed hadron energy, $E_{H}^{*}$, scaled down by the beam energy $\sqrt{s} / 2$. The variable $\rho$, appearing in (2), is its partonic equivalent. Within this production mechanism, the observed hadron $H$ is generated by the fragmentation of the final state parton $l$, and for this reason we address it as central. The amplitudes squared [21], however, are singular when the transverse momentum of the final state parton vanishes. In such configurations, the parent parton $l$ of the observed hadron $H$ is collinear either to the incoming parton $i$ or $j$. As these phase space regions are approached, perturbation theory loses its predictivity. This class of collinear singularities escape the usual renormalisation procedure which amounts to reabsorb collinear divergences into a redefinition of bare parton distribution and fragmentation functions. Such singularities are likely to 


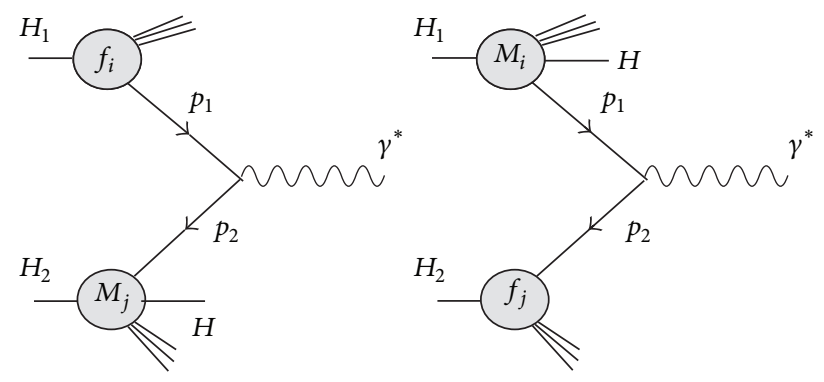

Figure 2: Pictorial representation of the parton model formula (4) for the associated production of a Drell-Yan pair and a particle in the target fragmentation regions.

appear in every fixed order calculation in the same kinematical limits spoiling the convergence of the perturbative series. In $[19,20]$ a generalised procedure for the factorisation of such additional collinear singularities is proposed. The latter is the same as the one proposed in Deep Inelastic Scattering [22] where the same singularities pattern is also found, confirming the universality of collinear radiation between different hard processes. Such a generalised collinear factorisation makes use of fracture functions. These distributions obey DGLAP-type evolution equations which contain an additional inhomogeneous term resulting from the subtraction of collinear singularities in the target fragmentation region $[6,22]$. Such equations allow us to resume the corresponding large logarithmic corrections to all orders in perturbation theory. Bare fracture functions, $M_{i}^{H / H_{1}}(x, z)$, describe the hadronization of the spectators system in hadron-induced reactions. They express the conditional probability to find a parton $i$ initiating the hard scattering while an hadron $H$ is produced with fractional momentum $z$ in the target fragmentation region of the incoming hadron $H_{1}$.

The use of fracture functions allows for particles production already to $\mathcal{O}\left(\alpha_{s}^{0}\right)$, since the hadron $H$ can be nonpertubatively produced by a fracture function itself. Therefore, the lowest order parton model formula can be symbolically written as

$$
\begin{aligned}
\frac{d \sigma^{H, T,(0)}}{d Q^{2} d z} & \propto \sum_{i, j} \int \frac{d x_{1}}{x_{1}} \int \frac{d x_{2}}{x_{2}} \\
\cdot & {\left[M_{i}^{[1]}\left(x_{1}, z\right) f_{j}^{[2]}\left(x_{2}\right)+M_{i}^{[2]}\left(x_{2}, z\right) f_{j}^{[1]}\left(x_{1}\right)\right] } \\
\cdot & \frac{d \widehat{\sigma}^{i j \rightarrow \gamma^{*}}}{d Q^{2}}
\end{aligned}
$$

and it is sketched in Figure 2. The superscripts in (4) indicate from which incoming hadron, $H_{1}$ or $H_{2}$, the outgoing hadron $H$ is produced through a fracture function. In order to complete the calculation to $\mathcal{O}\left(\alpha_{s}\right)$ accuracy we should consider higher order corrections to (4). Since in this case the hadron $H$ is already produced by fracture functions, final state parton radiation should be integrated and the resulting contribution added to virtual corrections. One of

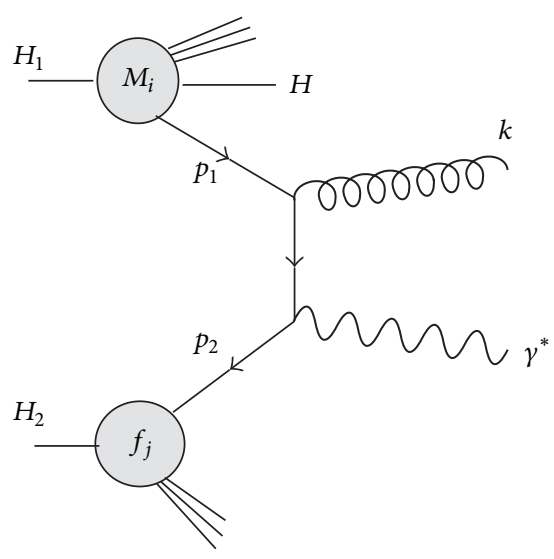

FIgURE 3: Example of diagram contributing to $\mathcal{O}\left(\alpha_{s}\right)$ corrections in the target fragmentation region (5).

the contributing diagrams is depicted in Figure 3. The general structure of these terms is

$$
\begin{aligned}
\frac{d \sigma^{H, T,(1)}}{d Q^{2} d z} \propto \sum_{i, j} \int \frac{d x_{1}}{x_{1}} \int \frac{d x_{2}}{x_{2}} \\
\cdot\left[M_{i}^{[1]}\left(x_{1}, z\right) f_{j}^{[2]}\left(x_{2}\right)+M_{i}^{[2]}\left(x_{2}, z\right) f_{j}^{[1]}\left(x_{1}\right)\right] \\
\cdot \frac{d \hat{\sigma}^{i j \rightarrow(l) \gamma^{*}}}{d Q^{2}} .
\end{aligned}
$$

We refer to them as to the target fragmentation contributions. Their calculation is, apart from minor differences in kinematics, completely analogous to the one of the inclusive Drell-Yan case. The factorisation procedure, first elaborated in [22] in the context of SIDIS, amounts to substitute in (4) the bare fracture and parton distributions functions with their renormalised version $[19,20]$. Renormalised parton distributions and fracture functions homogeneous terms do cancel, as in the inclusive Drell-Yan case, all singularities present in (5). The additional singularities in (2) are cancelled by the combination of parton distributions and fracture functions inhomogeneous renormalisation terms. Adding all the various contributions, the resulting hadron- $p_{t}$ integrated cross section, up to order $\mathcal{O}\left(\alpha_{s}\right)$, is then infrared finite $[19,20]$ and can be symbolically written as

$$
\begin{aligned}
& \frac{d \sigma^{H}}{d Q^{2} d z} \\
& =\frac{\sigma_{0}}{N_{c} s} \sum_{i, j}\left[M_{i}^{[1]} \otimes f_{j}^{[2]}+M_{i}^{[2]} \otimes f_{j}^{[1]}\right]\left(1+\frac{\alpha_{s}}{2 \pi} C^{i j}\right) \\
& \quad+\frac{\sigma_{0}}{N_{c} s} \frac{\alpha_{s}}{2 \pi} \sum_{i, j, l} f_{i}^{[1]} \otimes f_{j}^{[2]} \otimes D^{H / l} \otimes K_{l}^{i j},
\end{aligned}
$$

where $\sigma_{0}=4 \pi \alpha_{e m}^{2} / 3 Q^{2}$ and $N_{c}$ is the number of colors. We refer to the previous equation as to the collinear factorisation formula for the process under study. The next-toleading order coefficients $C^{i j}$ and $K_{l}^{i j}$ have been calculated 
in [20], making the whole calculation ready for numerical implementation.

We stress, however, that our ability to consistently subtract collinear singularities in such a semi-inclusive process is a necessary but not sufficient condition for factorisation to hold in hadronic collisions. The one-loop calculation outlined above in fact does involve only the so-called active partons. It completely ignores multiple soft parton exchanges between active and spectators partons, whose effects should be accounted for in any proof of QCD factorisation. Therefore, there is no guarantee that fracture functions extracted from SIDIS can be successfully used to describe forward or backward particle production in hadronic collisions. Reversing the argument, such a comparison may instead offer new insights into nonperturbative aspects of QCD and to the breaking of factorisation.

\section{Single Hard Diffraction at AFTER@LHC}

As an application of the formalism presented in the previous sections we will consider single hard diffractive production of a Drell-Yan pair:

$$
p_{1}\left(P_{1}\right)+p_{2}\left(P_{2}\right) \longrightarrow p(P)+\gamma^{*}(q)+X,
$$

where we have indicated in parentheses the four momenta of the relevant particles. We present in the following cross sections differential in the virtual photon variables. The subsequent decay of the virtual photon into a lepton pair can be easily included so that realistic cuts on leptons rapidity and transverse momentum can then be applied. We consider the AFTER@LHC kinematic setting in which a $7 \mathrm{TeV}$ proton beam collides on a fixed target proton leading to a centre-ofmass energy of $\sqrt{s}=115 \mathrm{GeV}$. We consider the projectile proton $p_{1}$ moving in the positive $z$ direction and $p_{2}$ at rest in the laboratory. The diffractively produced proton $p$ has in general almost the incoming projectile proton $p_{1}$ energy and very small transverse momentum as measured with respect to the collision axis. The detection of such fast protons will in general require the installation of forward proton taggers. The lepton pair instead will be measured by the main AFTER@LHC detector. This kinematical configuration is pictorially represented in the right plot of Figure 2 .

Diffractive processes have been intensively analysed in DIS at HERA ep collider, revealing their leading twist nature. From scaling violations of the diffractive structure functions $[7,10,11]$ and dijet production in the final state $[8,9]$ quite precise diffractive parton distributions functions (dPDFs) have been extracted from HERA data, which parametrise the parton content of the color singlet exchanged in the $t$-channel. The comparison of QCD predictions for single diffractive hard processes based on diffractive parton distributions measured at HERA against data measured at Tevatron $[16,17]$ $(\sqrt{s}=1.96 \mathrm{TeV})$, adopting a factorised ansatz as in (4), has indeed revealed that these processes are, not unexpectedly $[14,15]$, significantly suppressed in hadronic collisions. This conclusion persists even after the inclusion of higher order QCD corrections [23]. Complementing these results with the forthcoming ones from LHC at higher centre-of-mass energies $(\sqrt{s}=13 \mathrm{TeV})$ and the ones from AFTER@LHC at $\sqrt{s}=115 \mathrm{GeV}$ will give insight into the energy dependence of the so-called rapidity gap survival (RGS) probability in a wide range in $\sqrt{s}$. Since the theoretical computation of the RGS factor is highly model dependent, we decided not to include it in our predictions. Therefore, the latter must be considered as upper bounds.

Diffractive parton distributions $f_{i}^{D}$ are in general protonto-proton fracture functions $M_{i}$. They depend upon the final state proton fractional energy loss, $x_{\mathrm{IP}}=1-z$ with $z$ given in (3), the fractional momentum of the interacting parton with respect to the pomeron momentum, $\beta=x / x_{\mathrm{IP}}$ and the virtuality $Q^{2}$. In general fracture functions may depend also upon the invariant momentum transfer $t=\left(P-P_{1}\right)^{2}$ at the proton vertex [24]. In all diffractive structure functions measurements at HERA, out of which DPDFs are determined, $t$ is integrated up to some $t^{\max } \ll Q^{2}$. In this case dPDFs obey ordinary DGLAP evolution equations [25] as their extended, $t$-dependent version [24]. In the present paper we use dPDFs form [7] which are defined by $|t|<1 \mathrm{GeV}^{2}$. Since they are extracted from large rapidity gap data where the proton is not directly measured, they contain a contribution (23\%) from the so-called proton dissociation contribution. In order to use $\mathrm{dPDFs}$ in the present context we first note that

$$
M_{i}\left(x_{1}, z, Q^{2}\right)=x_{\mathrm{IP}}^{-1} f_{i}^{D}\left(\beta, x_{\mathrm{IP}}, Q^{2}\right) .
$$

The extra factor $x_{\mathrm{IP}}^{-1}$ comes from the Jacobian of the change $x_{1}=\beta x_{\text {IP }}$. We then rearrange (4) in terms of new variables obtaining

$$
\begin{aligned}
& \frac{d \sigma^{D}}{d Q^{2} d x_{\mathrm{IP}}} \\
& =\frac{\sigma_{0}}{N_{c} \mathcal{s}} \int_{\tau / x_{\mathrm{IP}}}^{1} \frac{d \beta}{\beta} \sum_{q, \bar{q}} e_{q}^{2} x_{\mathrm{IP}}^{-1} f_{q}^{D}\left(\beta, x_{\mathrm{IP}}, \mu_{F}^{2}\right) f_{\bar{q}}\left(\frac{\tau}{\beta x_{\mathrm{IP}}}, \mu_{F}^{2}\right),
\end{aligned}
$$

with $\tau=Q^{2} / s$. For simplicity we consider here leading order formulas but the extension to higher order is straightforward. In (9) we use parton distribution functions from [26]. We show explicitly the dependence of fracture and parton distributions functions upon the factorisation scale, $\mu_{F}^{2}$. Predictions are obtained with this scale set to $\mu_{F}^{2}=Q^{2}$. Theoretical errors associated with higher order corrections are instead estimated varying such scale in the range $\mu_{F}^{2}=$ $1 / 4 Q^{2}$ and $\mu_{F}^{2}=4 Q^{2}$.

In Figure 4 we present predictions for the $x_{\text {IP }}$ distribution. In left plot we consider a Drell-Yan pair of mass $Q^{2}=$ $100 \mathrm{GeV}^{2}$. The distribution shrinks as lower $x_{\mathrm{IP}}$-values are approached whereas, from hard diffraction at HERA, it is well known that diffractive cross sections rise as an inverse power of $x_{\text {IP }}$. Such an effect therefore is then attributed to phase space threshold effects. The Drell-Yan invariant mass constraint can be rewritten in the diffractive case as $Q^{2}=$ $\beta x_{\mathrm{IP}} x_{2}$ s, which can be cast (for $\beta \rightarrow 1$ and $x_{2} \rightarrow 1$ ) in upper bound on the invariant mass $Q^{2}<x_{\mathrm{IP}} s$ at fixed $x_{\mathrm{IP}}$ and $s$. This hypothesis is further supported in the right plot of Figure 4, where differential distributions are presented for three values of $Q^{2}$. The lowest values of $x_{\text {IP }}$ are then accessed 


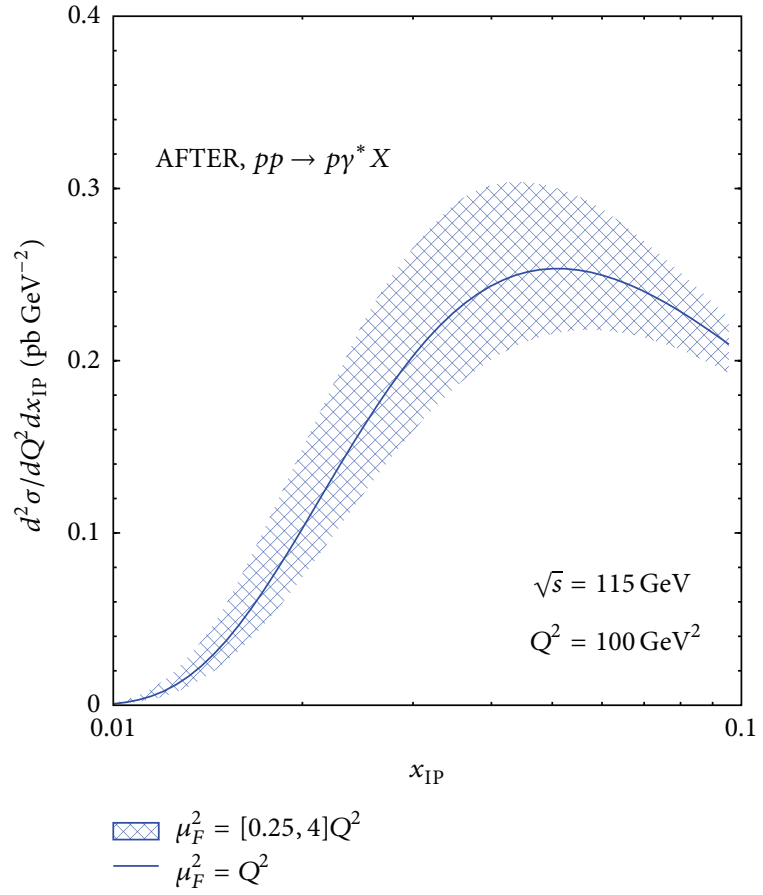

(a)

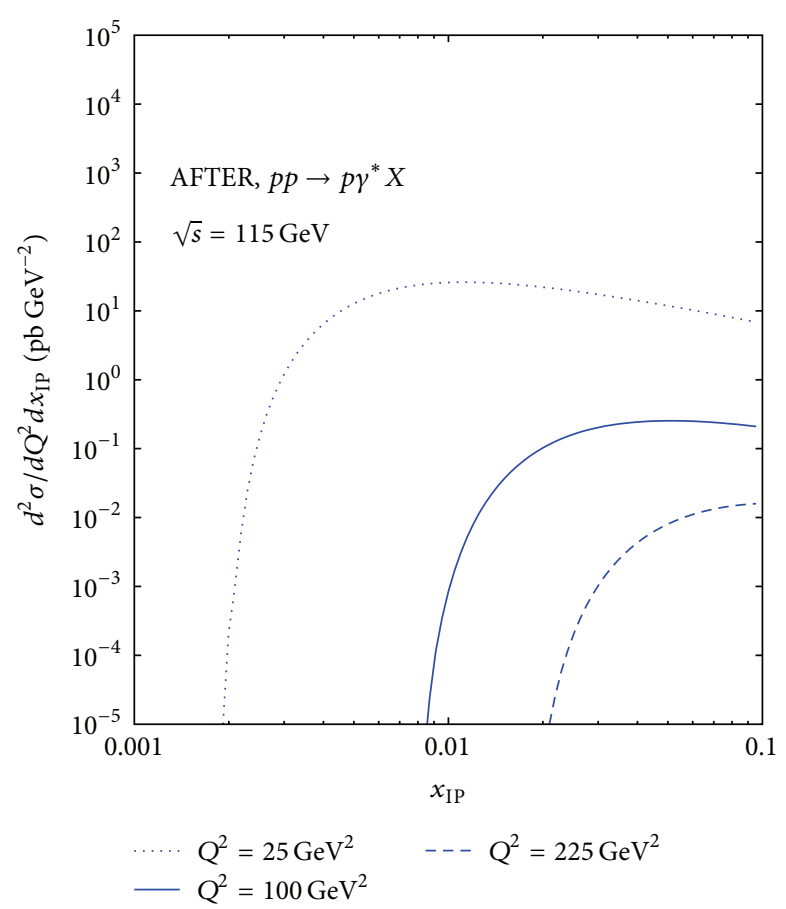

(b)

Figure 4: (a) Double differential cross sections for the production of a Drell-Yan pair of mass $Q^{2}=100 \mathrm{GeV}^{2}$. Blue error bands represent theoretical errors estimation, as described in the text. (b) Double differential cross sections for three different invariant masses.

only by lowering the invariant mass of the pair. We note that, even considering the maximum value of $x_{\mathrm{IP}}=0.1$, single diffractive production of $W^{ \pm}$and $Z$ is beyond the kinematic reach at AFTER@LHC. In the left panel of Figure 5 we present the prediction for the $Q^{2}$ distribution at a fixed value of $x_{\mathrm{IP}}=0.05$. The cross section, as expected, is fast falling as an inverse power of $Q^{2}$. The $Q^{2}$ distribution is particularly instructive since it allows us to study the possible dependence of the RGS factor on $Q^{2}$ and therefore to get an insight into the underlying dynamics. In the right panel of Figure 5 we present the $Q^{2}$-differential cross section multiplied by a factor $Q^{4}$, which compensates the fast fall off of the electromagnetic cross section so that all the $Q^{2}$ dependence is accounted for by that of fracture and parton distributions. These curves and the corresponding slopes, however, can not be readily interpreted as genuine results of QCD evolution of fracture and parton distributions functions because of the threshold effect mentioned above appearing at such moderate values of $\sqrt{s}$.

By changing variable from $\beta$ to the virtual photon centreof-mass rapidity, $y^{\mathrm{CM}}$,

$$
\begin{gathered}
\beta=\frac{\sqrt{\tau}}{x_{\mathrm{IP}}} e^{y^{\mathrm{CM}}}, \\
x_{2}=\sqrt{\tau} e^{-y^{\mathrm{CM}}},
\end{gathered}
$$

equation (9) can be further manipulated to give the threedifferential cross section:

$$
\begin{aligned}
& \frac{d \sigma^{D}}{d Q^{2} d x_{\mathrm{IP}} d y^{\mathrm{CM}}} \\
& \quad=\frac{\sigma_{0}}{N_{c} s} \sum_{q, \bar{q}} e_{q}^{2} x_{\mathrm{IP}}^{-1} f_{q}^{D}\left(\beta, x_{\mathrm{IP}}, \mu_{F}^{2}\right) f_{\bar{q}}\left(x_{2}, \mu_{F}^{2}\right) .
\end{aligned}
$$

The rapidity range for diffractive Drell-Yan production reads

$$
\ln \sqrt{\tau}<y^{\mathrm{CM}}<\ln \frac{\sqrt{\tau}}{x_{\mathrm{IP}}}
$$

which, as expected, turns out to be asymmetric given the kinematic constraint $x_{1}<x_{\text {IP }}$. The rapidity range for the inclusive Drell-Yan case is recovered simply setting $x_{\mathrm{IP}}=1$. The rapidity distribution is particularly sensitive to the shape of the diffractive parton distributions. This distribution will be useful to investigate any possible kinematic dependence of the RGS factor. In the left panel of Figure 6 we present the centre-of-mass rapidity distribution at fixed $Q^{2}=100 \mathrm{GeV}^{2}$ and $x_{\mathrm{IP}}=0.05$. In this frame the distribution is shifted at negative values of $y^{\mathrm{CM}}$. Therefore, on average, the parton originating from the target proton carries more momentum than the one originating from the pomeron. Since the rapidity 


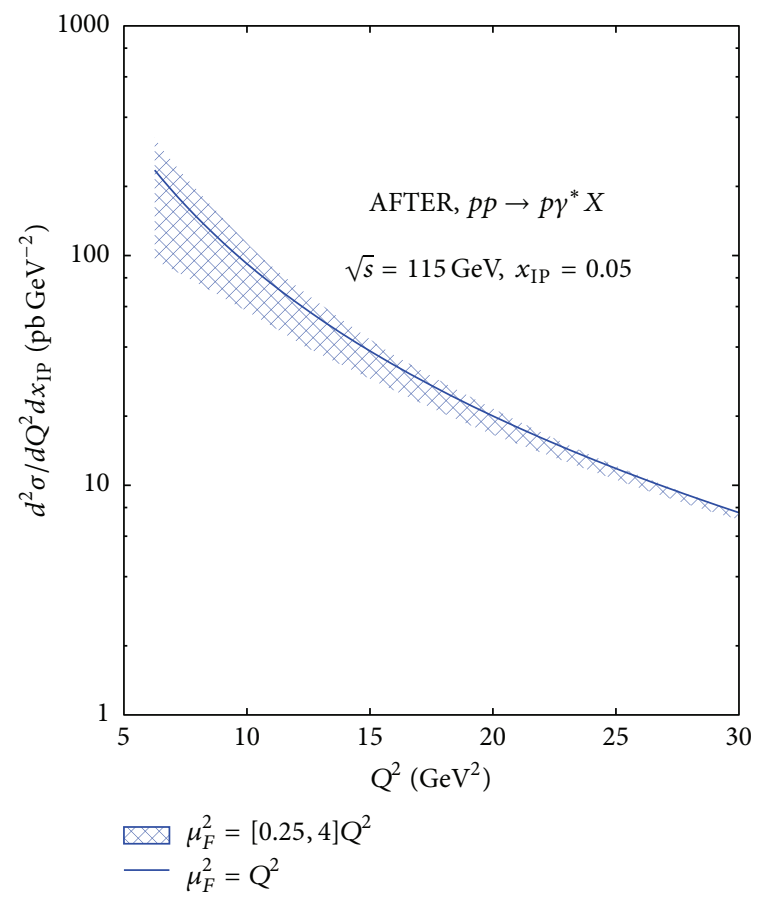

(a)

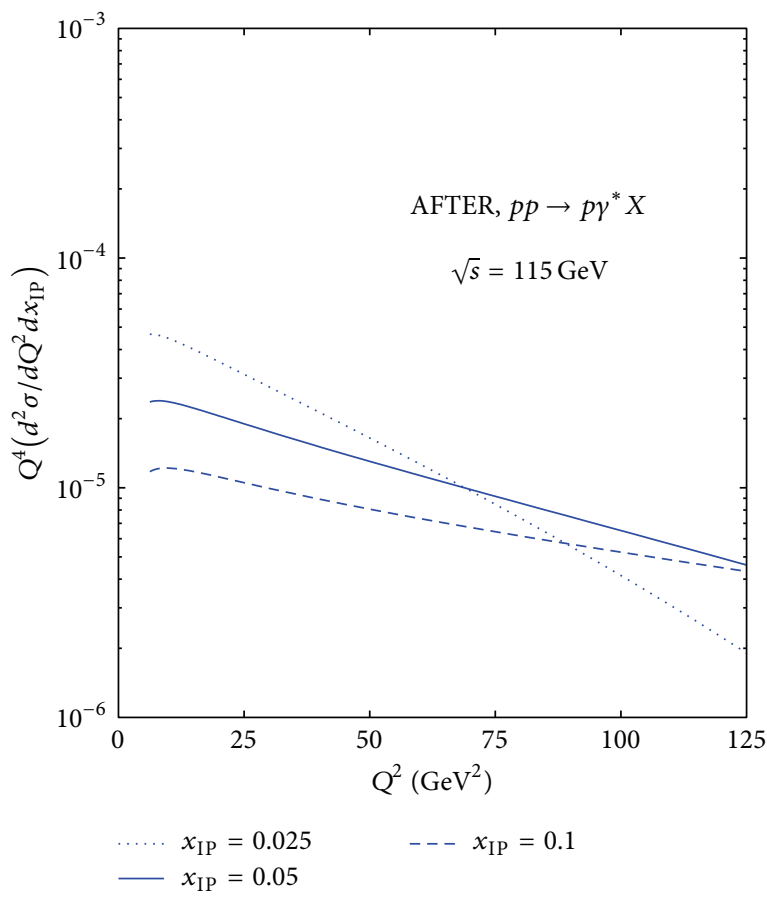

(b)

FIGURE 5: (a) Double differential cross sections for the production of a Drell-Yan pair at $x_{\mathrm{IP}}=0.05$. Blue error bands represent theoretical errors estimation, as described in the text. (b) Double differential cross sections times $Q^{4}$ for three different $x_{\mathrm{IP}}$-values.

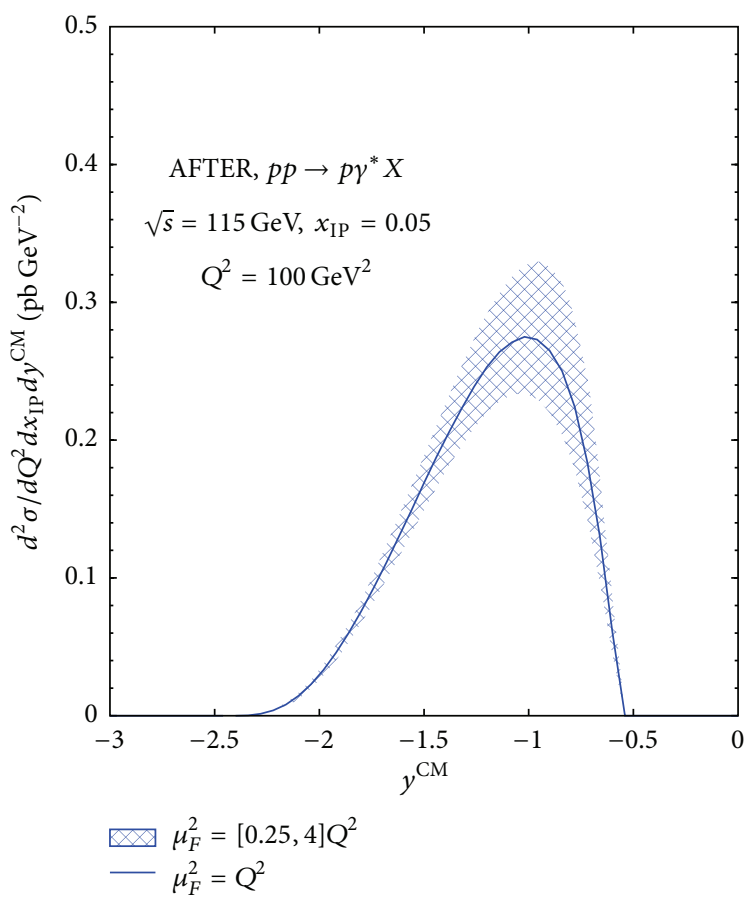

(a)

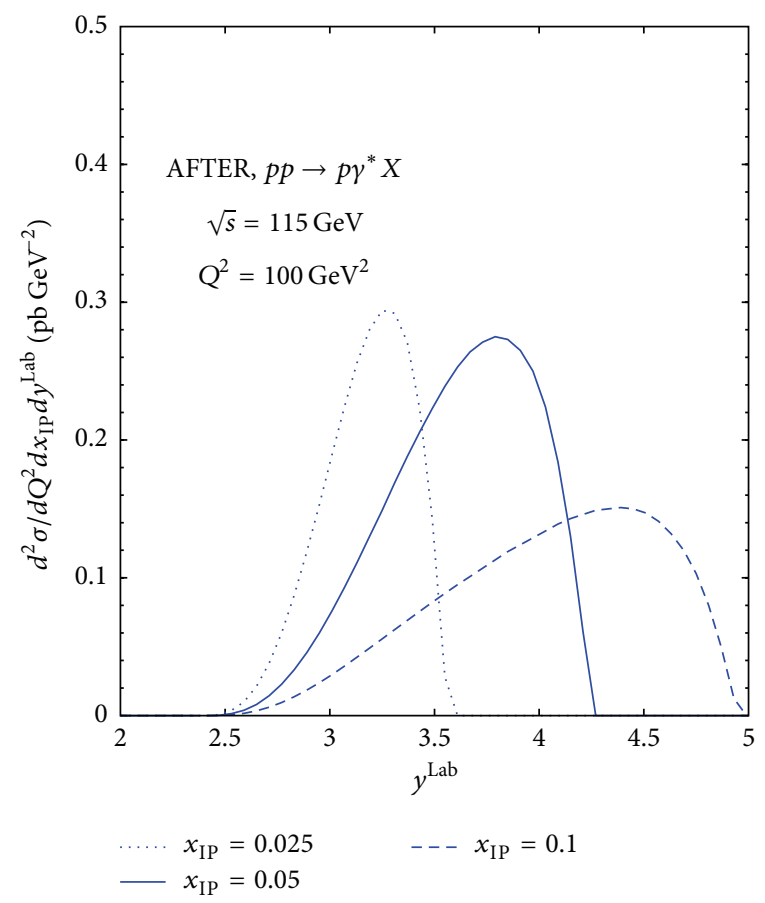

(b)

FIGURE 6: (a) Triple differential cross sections for the production of a Drell-Yan pair at $x_{\text {IP }}=0.05$ and of mass $Q^{2}=100 \mathrm{GeV}^{2}$. Blue error bands represent theoretical errors estimation, as described in the text. (b) Triple differential cross sections for three different $x_{\text {IP }}$-values. 


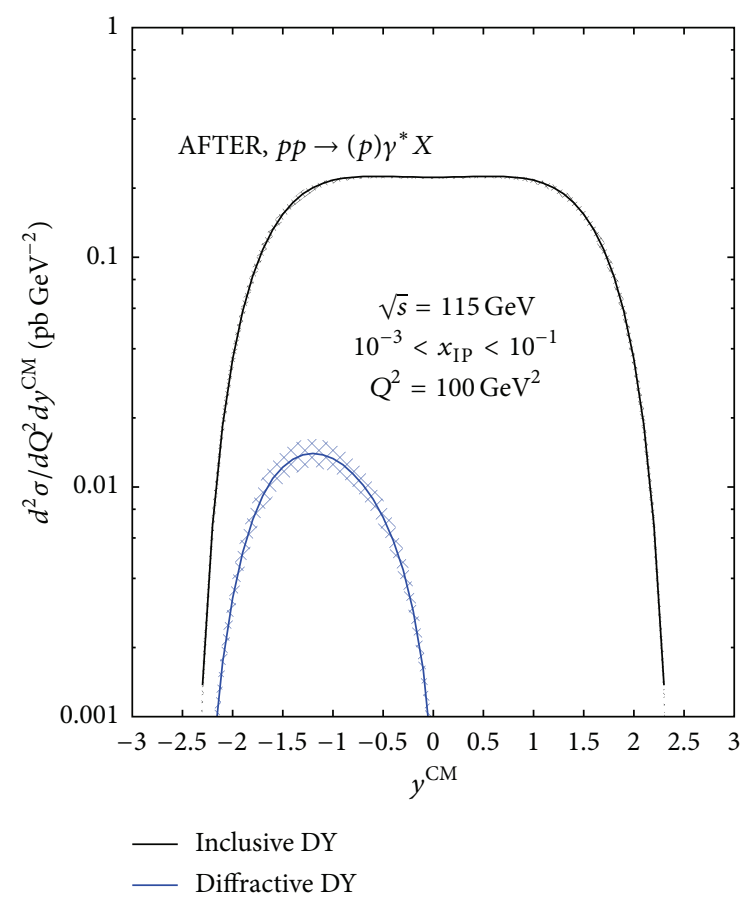

(a)

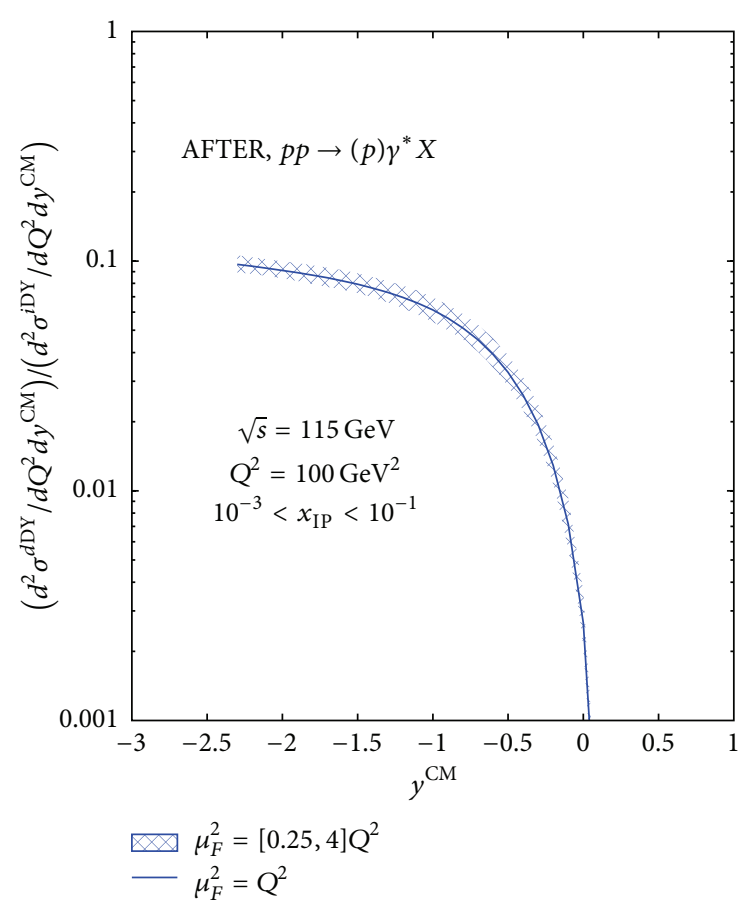

(b)

Figure 7: (a) Rapidity distributions for inclusive and diffractive Drell-Yan of mass $Q^{2}=100 \mathrm{GeV}^{2}$. Blue error bands represent theoretical errors estimation, as described in the text. (b) Diffractive to inclusive Drell-Yan rapidity distributions ratio.

is additive under boost along the collision axis we may easily boost the $y^{\mathrm{CM}}$ to the laboratory frame by using

$$
y^{\mathrm{Lab}}=\frac{1+c}{1-c}+y^{\mathrm{CM}} \quad \text { with } c=\sqrt{1-\frac{4 m_{p}^{2}}{s}}
$$

with $m_{p}$ the proton mass. In the AFTER@LHC kinematics this implies a rapidity shift $\Delta y=y^{\mathrm{Lab}}-y^{\mathrm{CM}}=4.8$. The rapidity distributions in the laboratory frame for a DrellYan pair of mass $Q^{2}=100 \mathrm{GeV}^{2}$ and for three different $x_{\text {IP }}$ values are presented in the right panel of Figure 6 . One may notice from the plot that for increasing $x_{\text {IP }}$ the DrellYan pair spans a wider rapidity range and the corresponding spectrum is increasingly more forward. It might be useful to discuss the single diffractive Drell-Yan pair production in conjunction with the analogous inclusive process. Such a comparison is presented for the centre-of-mass rapidity distributions in the left plot of Figure 7 for a common DrellYan pair of mass squared $Q^{2}=100 \mathrm{GeV}^{2}$ and, for the diffractive case, integrated in the range $10^{-3}<x_{\text {IP }}<$ $10^{-1}$. The rapidity distribution in the single diffractive case is strongly asymmetric whereas in the inclusive case it is symmetric around $y^{\mathrm{CM}}=0$. This effect is primarily due to the different kinematics of the two processes and to the different fractional momentum distributions between parton and fracture distributions. In the right plot of Figure 7 the ratio between the two previous distributions is presented. The ratio gives direct information on the suppression factor between the single diffractive to the inclusive process, assuming a factorised expression for the former (11). Such a ratio might be convenient from the experimental side since many lepton detection systematics will cancel. On the theoretical one it is expected to be more stable against the inclusion of higher order corrections. In the present case, for example, the factorisation scale is simultaneously varied both on the numerator and denominator resulting in a reduced theoretical error band with respect to the one obtained for absolute cross sections.

We wish to end this section with a brief overview of other possible applications of the proposed formalism. A completely analogous program can be performed for the associated production of forward neutron and a Drell-Yan pair, $p+p \rightarrow n+\gamma^{*}+X$. The production of forward neutron in DIS at HERA has shown a leading twist nature. From scaling violations of the semi-inclusive neutron structure functions a set of proton-to-neutron fracture functions has been extracted from data in [12] which can be used to predict forward neutron rate in hadronic collisions. As in the case of hard diffraction, both physics programs would highly benefit from the installation of a dedicated instrumentation for the measurements of fast neutrons and protons quite close to the beam axis. Measurements in the forward region, although problematic experimentally, give in fact direct access to the study of the beam fragmentation region.

As a third application we consider hyperon production associated with a Drell-Yan pair, $p+p \rightarrow V+\gamma^{*}+X$, where $V$ generically indicates either a $\Lambda^{0}$ or $\bar{\Lambda}^{0}$ hyperon. At very low transverse momentum, the $\Lambda^{0}$ longitudinal momentum 
spectrum should show a significant leading particle effect, which can be predicted, assuming factorisation as in $d \sigma^{H, T}$, by the proton-to-lambda fracture functions set obtained from a fit to SIDIS data in [13]. On the other hand, the $\bar{\Lambda}^{0}$ spectrum in the same kinematical conditions should instead show almost no leading particle effect, giving access to the proton-to- $\bar{\Lambda}^{0}$ fracture functions. We note, in general, that the particle-to-antiparticle fracture function is indeed an interesting and almost unknown distribution. On the other hand, if one considers $\Lambda^{0}$ or $\bar{\Lambda}^{0}$ at sufficiently large transverse momentum, their combined analysis, described by $d \sigma^{H, C}$, should allow for the investigation of parton hadronisation into hyperons in the QCD vacuum as parametrised by fragmentation functions.

As a last application we consider the associated production of one particle and a Drell-Yan pair in the context of multiparton interactions. The latter process has already been used to investigate the contamination of the so-called underlying event [27] to jet observable and has been successfully used to study underlying event properties [28]. If the detected hadron is measured at sufficiently large transverse momentum, the latter constitutes a natural infrared regulator for the partonic matrix elements. In this kinematical conditions we also expect a rather small contribution from fracture functions. Therefore, the central term, $d \sigma^{H, C,(1)}$, can be used to estimate the single parton scattering contribution to the process. The latter might be considered as the baseline to study the double (or multiple) parton scattering contributions to the same final state, where, for example, the primary scatterer produces a Drell-Yan pair while the secondary one produces the detected hadron $H$.

\section{Conclusions}

We have briefly reviewed a perturbative approach to single particle production associated with a Drell-Yan pair in hadronic collisions. On the theoretical side we have shown that the introduction of fracture functions allows a consistent factorisation of new class of collinear singularities arising in this type of processes. The factorisation procedure coincides with the one used in DIS confirming, as expected, the universal structure of collinear singularities and supports the proposed collinear factorisation formula. On the phenomenological side we have outlined some areas in which the formalism can be fully tested. In particular, focusing on the AFTER@LHC kinematical range, we have presented numerical predictions for the single diffractive production of virtual photons. The study of such a process might improve our understanding of nonperturbative aspects of QCD and it allows one to explore in detail the nature of factorisation breaking at intermediate energies.

\section{Conflict of Interests}

The author declares that there is no conflict of interests regarding the publication of this paper.

\section{References}

[1] M. Basile, G. Cara Romeo, L. Cifarelli et al., "The 'leading'particle effect in hadron physics," Nuovo Cimento A, vol. 66, no. 2, pp. 129-163, 1981.

[2] J. C. Collins, "Proof of factorization for diffractive hard scattering," Physical Review D, vol. 57, no. 5, pp. 3051-3056, 1998.

[3] J. C. Collins, "Erratum: proof of factorization for diffractive hard scattering [Phys. Rev. D 57, 3051 (1998)]," Physical Review D, vol. 61, no. 1, Article ID 019902, 1 page, 1999.

[4] J. C. Collins, "Factorization in hard diffraction," Journal of Physics G: Nuclear and Particle Physics, vol. 28, no. 5, pp. 10691077, 2002.

[5] M. Grazzini, L. Trentadue, and G. Veneziano, "Fracture functions from cut vertices," Nuclear Physics B, vol. 519, no. 1-2, pp. 394-404, 1998.

[6] L. Trentadue and G. Veneziano, "Fracture functions. An improved description of inclusive hard processes in QCD," Physics Letters B, vol. 323, no. 2, pp. 201-211, 1994.

[7] A. Aktas, V. Andreev, T. Anthonis et al., "Measurement and QCD analysis of the diffractive deep-inelastic scattering cross section at HERA," The European Physical Journal C-Particles and Fields, vol. 48, no. 3, pp. 715-748, 2006.

[8] H1 Collaboration, "Dijet cross sections and parton densities in diffractive DIS at HERA," Journal of High Energy Physics, vol. 2007, no. 10, article 042, 2007.

[9] ZEUS Collaboration, S. Chekanov, M. Derrick et al., "A QCD analysis of ZEUS diffractive data," Nuclear Physics B, vol. 831, no. 1-2, pp. 1-25, 2010.

[10] F. A. Ceccopieri and L. Favart, "Revisiting QCD fits in diffractive DIS," http://arxiv.org/abs/1205.6356.

[11] F. A. Ceccopieri and L. Favart, "QCD fits in diffractive DIS revisited," http://arxiv.org/abs/1110.4829.

[12] F. A. Ceccopieri, "QCD analysis of forward neutron production in DIS," The European Physical Journal C, vol. 74, article 3029, 2014.

[13] F. A. Ceccopieri and D. Mancusi, "QCD analysis of Lambda hyperon production in DIS target-fragmentation region," The European Physical Journal C, vol. 73, article 2435, 2013.

[14] A. Berera and D. E. Soper, "Diffractive jet production in a simple model with applications to DESY HERA," Physical Review D, vol. 50, article 4328, 1994.

[15] J. C. Collins, L. Frankfurt, and M. Strikman, "Diffractive hard scattering with a coherent pomeron," Physics Letters B, vol. 307, no. 1-2, pp. 161-168, 1993.

[16] CDF Collaboration, "Diffractive dijets with a leading antiproton in $\bar{p} p$ collisions at $\sqrt{s}=1800 \mathrm{GeV}$," Physical Review Letters, vol. 84 , p. $5043,2000$.

[17] T. Aaltonen, A. González, S. Amerio et al., "Diffractive $W$ and $Z$ production at the Fermilab Tevatron," Physical Review D, vol. 82, no. 11, Article ID 112004, 10 pages, 2010.

[18] S. J. Brodsky, F. Fleuret, C. Hadjidakis, and J. P. Lansberg, "Physics opportunities of a fixed-target experiment using LHC beams," Physics Reports, vol. 522, no. 4, pp. 239-255, 2013.

[19] F. A. Ceccopieri and L. Trentadue, "NLO semi-inclusive DrellYan cross-section in quantum chromodynamics as a factorization analyzer," Physics Letters B, vol. 668, no. 4, pp. 319-323, 2008.

[20] F. A. Ceccopieri, "Associated production of one particle and a Drell-Yan pair in hadronic collisions," Physics Letters B, vol. 703, no. 4, pp. 491-497, 2011. 
[21] G. Altarelli, R. K. Ellis, and G. Martinelli, "Large perturbative corrections to the Drell-Yan process in QCD," Nuclear Physics $B$, vol. 157, no. 3, pp. 461-497, 1979.

[22] D. Graudenz, "One-particle inclusive processes in deeply inelastic lepton-nucleon scattering," Nuclear Physics B, vol. 432, no. 1-2, pp. 351-373, 1994.

[23] M. Klasen and G. Kramer, "Survival probability for diffractive dijet production in $p \bar{p}$ collisions from next-to-leading order calculations," Physical Review D, vol. 80, Article ID 074006, 2009.

[24] G. Camici, M. Grazzini, and L. Trentadue, "Fracture functions and Jet Calculus," Physics Letters, Section B, vol. 439, no. 3-4, pp. 382-388, 1998.

[25] F. A. Ceccopieri and L. Trentadue, "A new fracture function approach to QCD initial state radiation," Physics Letters B, vol. 655, no. 1-2, pp. 15-25, 2007.

[26] A. D. Martin, R. G. Roberts, W. J. Stirling, and R. S. Thorne, "NNLO global parton analysis," Physics Letters B, vol. 531, no. 3-4, pp. 216-224, 2002.

[27] T. Affolder, H. Akimoto, A. Akopian et al., "Charged jet evolution and the underlying event in proton-antiproton collisions at 1.8 TeV," Physical Review D, vol. 65, no. 9, Article ID 092002, 22 pages, 2002.

[28] T. Aaltonen, J. Adelman, B. Álvarez González et al., "Studying the underlying event in Drell-Yan and high transverse momentum jet production at the Tevatron," Physical Review D, vol. 82, Article ID 034001, 2010. 

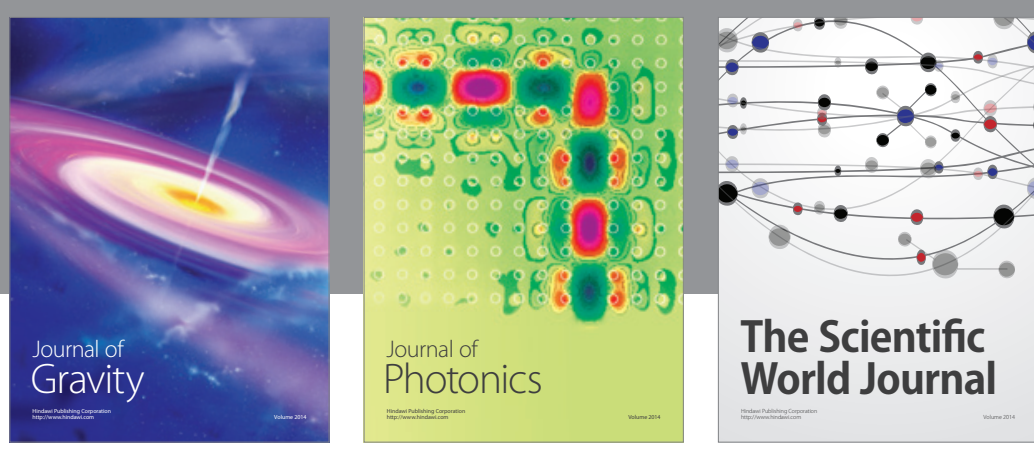

The Scientific World Journal
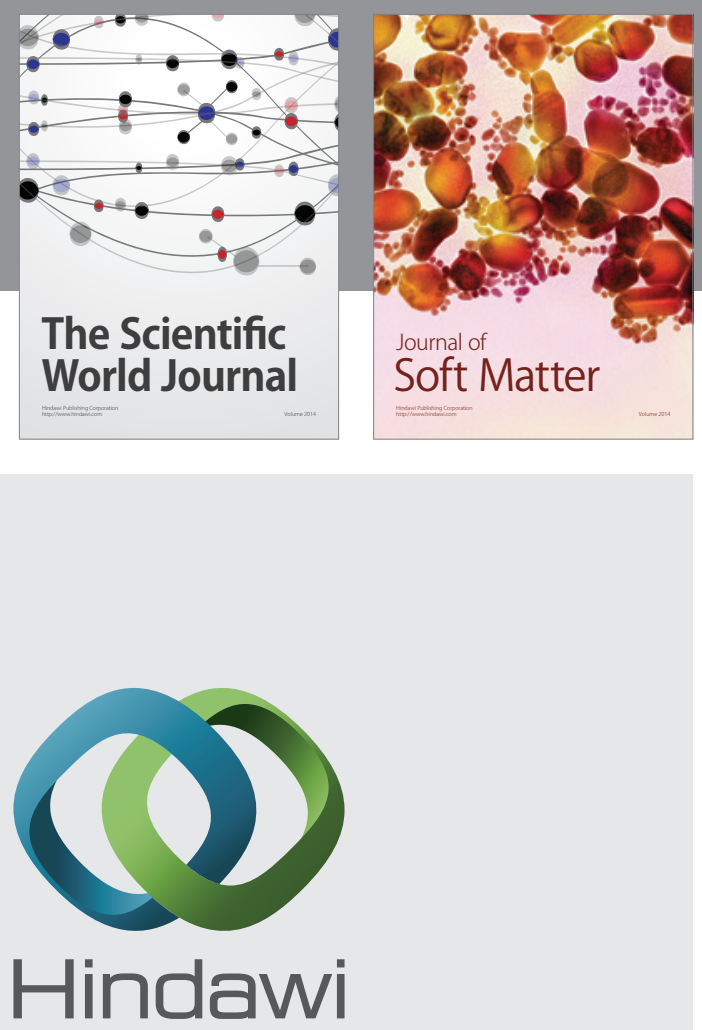

Submit your manuscripts at

http://www.hindawi.com

nternational Journal of

Statistical Mechanics
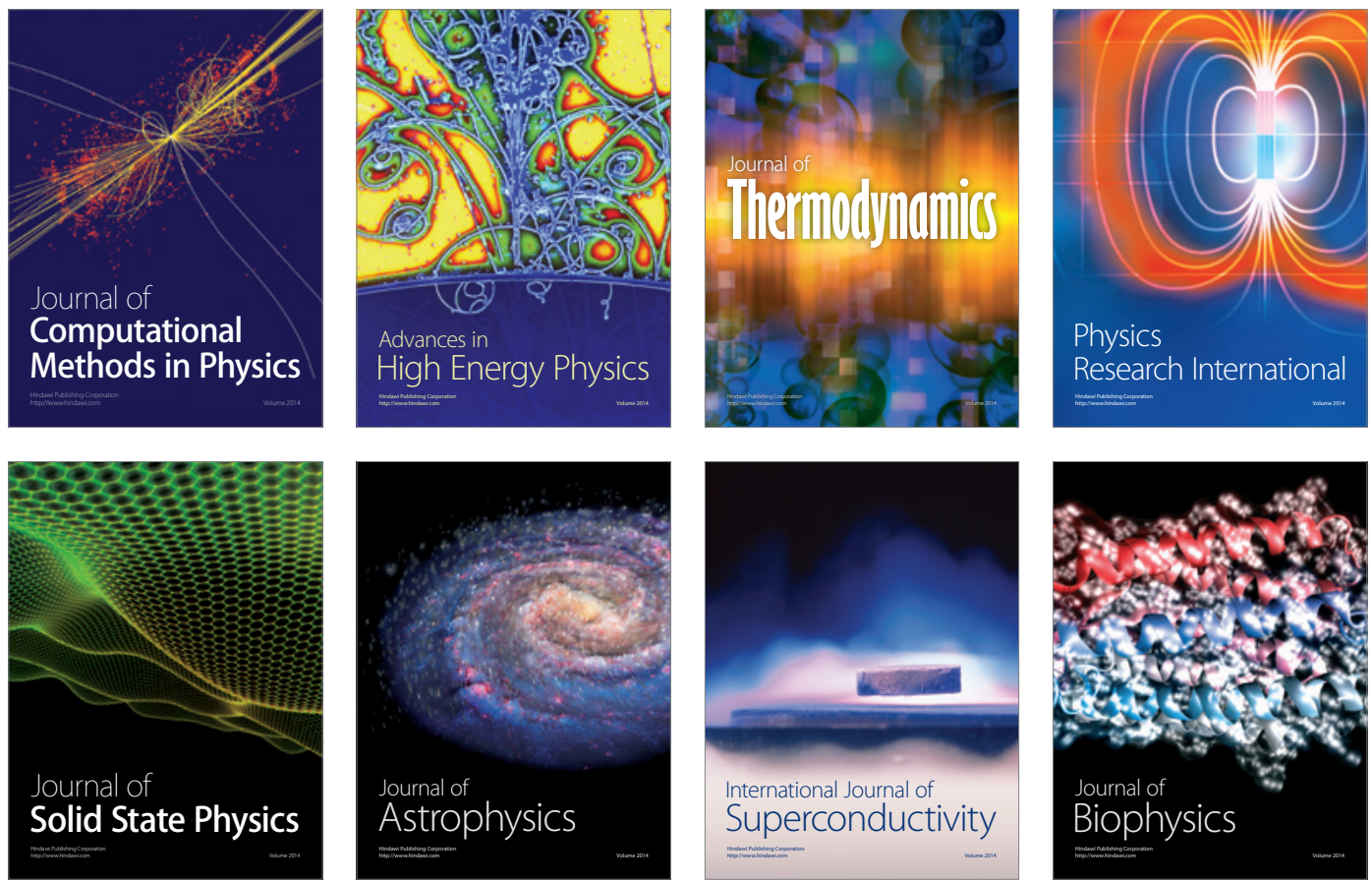
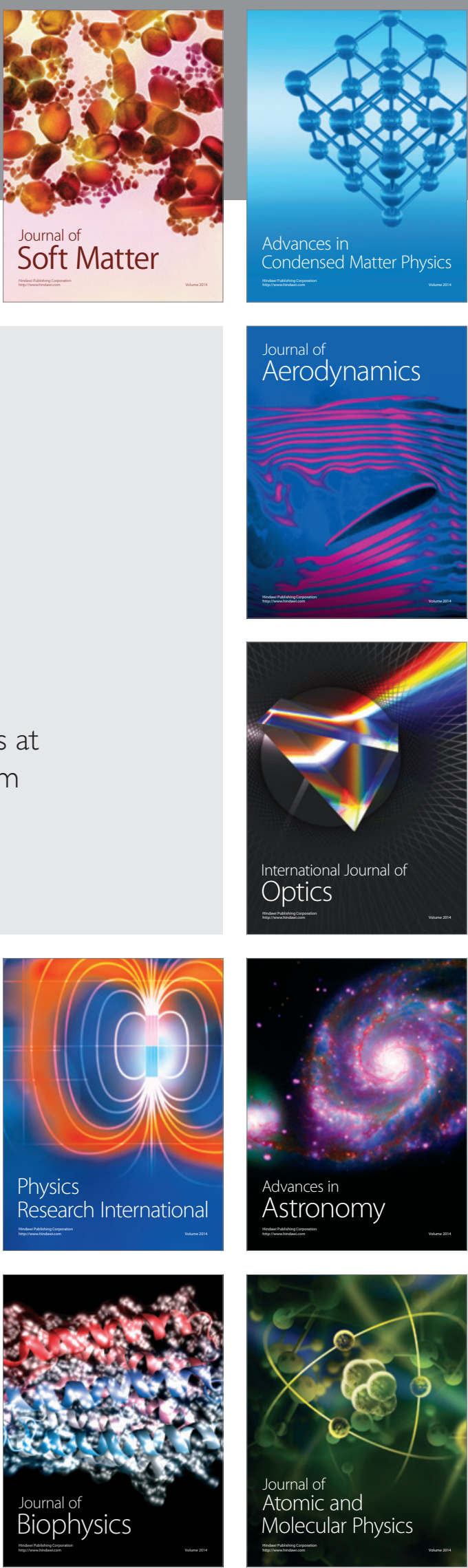